\title{
ESTRATEGIAS METACOGNITIVAS EN LA OPTIMIZACIÓN DEL APRENDIZAJE DE LOS MARCADORES DEL DISCURSO EN ESTUDIANTES UNIVERSITARIOS
}

\author{
María del Carmen Llontop Castillo \\ Universidad Nacional Federico Villarreal \\ llontopcastillo@gmail.com
}

\section{RESUMEN}

El objetivo de esta investigación, que constituye un resumen de la tesis doctoral Llontop (2015), consiste en evaluar en qué medida las estrategias metacognitivas optimizan significativamente el aprendizaje de los marcadores discursivos en estudiantes del Programa de Estudios Básicos de la Universidad Ricardo Palma en el año 2015. El diseño de la investigación fue experimental porque hubo manipulación de la variable independiente y en consecuencia modificación de la variable dependiente. Se define bajo el nivel cuasi experimental pretest - postest con grupo experimental y control; analizado mediante el enfoque mixto porque las conclusiones no solo se basan en datos cuantitativos de los test aplicados, sino también en datos cualitativos, por lo que también se diseñaron instrumentos de recolección de datos cualitativos. La muestra estuvo constituida por dos grupos de 27 estudiantes. Se aplicó un programa experimental que duró tres semanas donde los estudiantes del grupo experimental aplicaron estrategias metacognitivas en el aprendizaje de marcadores discursivos. Después del experimento se aplicó el post test para constrastar con los datos obtenidos en el pretest. Los resultados permitieron afirmar la hipótesis general y las específicas con resultados de P-Valor menor que el valor de alfa $\alpha=0.05$.

\section{PALABRAS CLAVE}

Marcadores discursivo/ Metacognición/ Estrategias de aprendizaje/ Adquisición/ extensión/ refinamiento.

\section{ABSTRACT}

The objective of this research, which is a summary of the doctoral thesis Llontop (2015), is to assess the extent metacognitive strategies significantly optimize learning discourse markers in Program students Basic Studies at the University 
Ricardo Palma in the 2015. The research design was experimental because there was manipulation independent variable and therefore modification of the dependent variable. It is defined under the quasi-experimental pretest level - posttest with experimental and control groups; analyzed by the mixed approach because the conclusions are based not only on quantitative data of the test applied, but also on qualitative data, so instruments qualitative data collection were also designed. The sample consisted of two groups of 27 students. An experimental three-week program where students in the experimental group applied metacognitive strategies in learning discourse markers applied. After the experiment the test was applied to post to contrast with data obtained in the pretest. The results allowed stating the general and specific hypotheses Results P-value less than the value of alpha $\alpha=0.05$.

\section{KEY WORDS}

Discourse markers/ Metacognition/ Learning strategies/ Acquisition/ Expansion/ refinement.

\section{INTRODUCCIÓN}

En la actualidad, la redacción de textos formales es considerada como una actividad muy compleja, porque consiste en plasmar por escrito las ideas buscando reflejar a través de las palabras todo el sentido que deseamos comunicar. La puesta en práctica de estrategias para mejorar la capacidad de procesamiento de información es de suma importancia sobre todo en las instituciones de educación superior, pero aún más las estrategias que permitan al estudiante aprender a redactar de manera segura y generando hábitos reguladores de la escritura. Así también, se sabe que el significado inferencial que un texto comunica radica sobre todo en el significado que aportan los enlaces, que en la bibliografía moderna se denominan marcadores discursivos. Son estos elementos lingüísticos los responsables de construir el significado inferencial de los textos y son de distinta tipología. Casualmente, porque de ellos depende el significado inferencial del texto, se consideran como los ordenadores de ideas en el texto, así como los organizadores y estructuradores de los elementos lingüísticos, cuando se disponen a comunicar; en este sentido es muy importante aprender a usarlos en la redacción. Se ha detectado que los estudiantes universitarios presentan el problema de no plasmar el contenido de un texto en forma coherente, sobre todo, por el uso inapropiado de los marcadores discursivos como elementos de cohesión pero al mismo tiempo como elementos que construyen el significado inferencial de textos del nivel universitario. Por esta razón esta investigación tiene como objetivo evaluar la optimización de las 
estrategias metacognitivas en el aprendizaje de los marcadores discursivos en una muestra de estudiantes universitarios. La hipótesis de investigación establece que las estrategias metacognitivas optimizan significativamente el aprendizaje de los marcadores discursivos de esos estudiantes, debido a que regulan todo tipo de pensamiento encaminado al aprendizaje autónomo de dichos elementos lingüísticos, los que darían la posibilidad de redactar el contenido lógico del texto en forma adecuada. Además, las estrategias metacognitivas son reguladoras en todo proceso de aprendizaje.

Esta investigación titulada Estrategias metacognitivas en la optimización del aprendizaje de los marcadores discursivos se ha desarrollado en una muestra conformada por dos grupos de investigación, el grupo control y el experimental, ambos extraídos como grupos naturales de la población de estudiantes matriculados en el Programa de Estudios Básicos de la Universidad Ricardo Palma durante el año 2015. Tiene como antecedentes inmediatos la investigación de Marín Aliaga, Flor de María que en el año 2010 sustentó la tesis de maestría en el Instituto para la Calidad de la Educación de la Universidad de San Martín de Porres, titulada Influencia de las estrategias metacognitivas en el desarrollo de la capacidad para solucionar problemas matemáticos; demostró que las estrategias metacognitivas son las más adecuadas para el área de ciencias debido a que influyen significativamente en el mejoramiento de la capacidad para solucionar problemas matemáticos; cuanto mejor se apliquen estas estrategias, mejor desarrollada estará la capacidad. De igual modo, la investigación presentada por Jacinto León, Antonio en el año 2009 también en la misma universidad, Estrategias metacognitivas y el logro de aprendizaje cognitivo biológico en alumnos de la Institución Educativa Manuel González Prada - Chimbote; demostró que existe relación entre las estrategias metacognitivas que aplican los estudiantes cuando aprenden biología, sobre todo porque esta especialidad requiere de mayor procesamiento cognitivo. Otros antecedentes importantes que permiten realizar la discusión en esta investigación, constituyen la investigación de Romero Díaz, Alejandra (2008) que en su tesis de maestría (USMP-ICED) demostró que existe relación significativa entre las Estrategias metacognitivas y el desarrollo de las habilidades de comprensión lectora en los niños de 5 años. Concluye que a mayores estrategias habrá mayor desarrollo de las habilidades de comprensión de textos, en consecuencia mejor significatividad de los resultados en los aprendizajes. Bara Soro, Pedro (2001) en su tesis doctoral Estrategias metacognitivas y de aprendizaje: estudio empírico sobre el efecto de la aplicación de un programa metacognitivo, y el dominio de las estrategias de aprendizaje en estudiantes de ESO, BUP y Universidad, pudo comprobar el importante papel de las estrategias en el proceso de aprendizaje que, dentro de la corriente cognitiva, persigue el objetivo de 
aprender a aprender, proporcionando al alumno las herramientas para que sea capaz de abordar la información y adquirir un conocimiento útil en múltiples situaciones. Este investigador aplicó un programa de estrategias metacognitivas en dos centros públicos de educación secundaria de Madrid a través de un diseño cuasi - experimental pretest - postest. Concluyó que existe buena influencia en la mejora del aprendizaje en distintas áreas del conocimiento. Finalmente, el antecedente constituido por la investigación de Nogueira da Silva, A. (2011) en su tesis titulada La enseñanza de los marcadores del discurso del español en relación con los géneros y secuencias textuales, sustentada en la Universidad de Salamanca y cuyo avance se encuentra publicado en la Revista Nebrija de Lingüística Aplicada (2010, Nro 9), no solo analiza la introducción de los marcadores discursivos en los materiales de español como lengua extranjera, sino que señala el supuesto de que el aprendizaje de los géneros y secuencias textuales puede beneficiarse del aprendizaje de los marcadores discursivos y viceversa. En la investigación de Bara (2001) se describen y analizan los marcadores discursivos dentro de un género textual producido por un aprendiz de español como lengua extranjera y propone dar relevancia al momento de elaborar materiales de enseñanza de lengua extranjera al aprendizaje de estas partículas considerando sobre todo sus valores semántico-pragmáticos y su magnitud en la construcción del discurso. Es un antecedente que evidencia que se deben aprender a usar los marcadores discursivos de manera significativa. Todos estos antecedentes evidencian la importancia de las estrategias metacognitivas y constituyen un referente para llevar a cabo la investigación pero en relación a los marcadores discursivos, permitirán ratificar la eficacia de las estrategias metacognitivas en todo proceso de aprendizaje, por lo que permiten establecer una validez externa.

La hipótesis de investigación presenta dos variables: Estrategias metacognitivas (Vi) y por otro lado Aprendizaje de los marcadores discursivos (Vd). Ha sido la variable independiente manipulada para poder evaluar la optimización que genera en el aprendizaje de los marcadores discursivos después de un cuasiexperimento utilizando un programa basado en estrategias metacognitivas que permitieron la planificación, el control y la evaluación del uso de los marcadores en los estudiantes del grupo experimental, para aprender a utilizarlos cuando se redacta un texto; por lo que permitió responder a la pregunta: ¿En qué medida las estrategias metacognitivas optimizan significativamente el aprendizaje de los marcadores discursivos en estudiantes del Programa de Estudios Básicos-URP en el año 2015?

Para lograr los objetivos se aplicó un pretest y un postest. Los resultados fueron procesados a través de distintas pruebas del SPSS 22 para muestras 
independiente y muestras emparejadas. Es decir permitió obtener los resultados de cada grupo (control y experimental) a nivel intragrupo y luego se obtuvieron resultados intergrupos, comparando resultados.

La investigación se desarrolla con un respaldo de bases teóricas, que dan sustento al análisis de los resultados tanto cualitativos como cuantitativos. Esta teoría se refiere a la metacognición en relación a su inclusión dentro del proceso de aprendizaje así como a la naturaleza de los marcadores discursivos como elementos lingüísticos responsables de la construcción del contenido lógico del mensaje textual. La actual teoría y su difusión permiten establecer que la investigación en el área de las estrategias de aprendizaje se está volviendo recurrente, sobre todo en aquellas dedicadas a mejorar las prácticas educativas que ejecutan los docentes y que buscan generar un mejor aprendizaje por parte de los estudiantes. Además, se sabe que las estrategias de aprendizaje consisten en procedimientos y recursos sobre todo cognitivos que los estudiantes utilizan cuando se disponen a aprender. Es así que Valle et al. (1998) establecen que " las estrategias de aprendizaje que los estudiantes ponen en marcha se encuentran muy relacionadas con los componentes cognitivos que influyen en el proceso de aprender (...) si asumimos la hipótesis que los motivos e intenciones del estudiante determinan (...) el tipo de estrategias que va a poner en marcha, ello implica que los mecanismos cognitivos que utilizan los sujetos para facilitar el aprendizaje dependen en gran medida de factores disposiciones y motivacionales" (p.53). De esta cita se establece que las estrategias que utilizan los estudiantes aseguran que lo que vaya a aprender tenga éxito y sea significativo. Además, debe concluirse que es definitivamente el componente cognitivo que utilizan los estudiantes cuando aprenden, por lo tanto las estrategias que deben fomentarse en una sesión de clase deben ser sobre todo de esta índole, estrategias cognitivas o metacognitivas. Por otro lado, Weinstein y Mayer (1986), en Valle et al. (1998), definen a las estrategias "...como conductas y pensamientos que un aprendiz utiliza durante el aprendizaje con la intención de influir en su proceso de codificación". (p.315). De esto se deduce que un aprendiz se orienta por las estrategias que utiliza, por lo tanto debe tener incorporadas las más adecuadas para el contenido, práctica o actitud que aprenderá.

El término metacognición, introducido en el campo de la psicología por John H. Flavell en la década de los 70 a través del estudio sobre la memoria, en el área de la lingüística, se ha denominado "metalenguaje" aludiendo a un lenguaje natural o formal que tiene por cometido describir el lenguaje. Por lo tanto la metacognición busca la regulación y organización de los procesos cognitivos que usamos cuando se realiza una actividad académica como el desarrollo de capacidades 
lingüísticas. Cuando se desea aprender, es importante que el estudiante conozca de manera consciente su procedimiento de aprendizaje y este conocimiento pueda ser controlado, es decir planificado, puesto en uso y evaluado para regularlo. Si el estudiante planifica, establece toda una serie de metas para la tarea que tiene que resolver, por lo tanto debe ser capaz también de predecir los resultados que obtendrá con lo que conoce estratégicamente. Es importante recalcar que como seres humanos poseemos muchas capacidades cognitivas por naturaleza, pero es la educación formal que las perfecciona y la vuelve explícitas.

El estudiante cuando se ve inmerso en un proceso de enseñanza-aprendizaje trae su capacidad metacognitiva natural, es tarea del docente aprovechar esto y volverla óptima; el docente debe fomentar las estrategias metacognitivas dentro de su sesión porque son las más ideales para que el estudiante aprenda con éxito porque el estudiante podrá controlar lo que hace, se dará cuenta si aprende o no y buscará darle solución a los problemas que se le puedan presentar, es decir regular lo que viene ejecutando.

En el fascículo 7 de la serie de Pedagogía que el Ministerio de Educación del Perú publicó sobre La comunicación, la cognición y la metacognición, se establece que los estudiantes más eficientes suelen ser aquellos que son conscientes de lo que saben y de lo que aún no domina; asimismo son aquellos que, frente a una situación nueva de aprendizaje o una dificultad, recurren a una serie de estrategias. Definitivamente un aprendizaje eficaz y duradero depende de una conciencia y control que los estudiantes hagan de sus procesos cognitivos, lo que implica que pedagógicamente se debe estimular la autonomía a partir de la toma de conciencia de cómo se produce su aprendizaje y qué deben hacer para optimizarlo. Esto supone que deben usar lo metacognitivo para regular su aprendizaje, porque vuelve al proceso más dinámico, creativo y personal. Por lo tanto, no basta solo que el docente sea un especialista en su materia o que utilice estrategias que motiven la resolución de una tarea sino que genere la autonomía del estudiante dentro de su sesión de aprendizaje; es decir, debe fomentar que el estudiante aprenda a aprender mientras va desarrollando su clase. Además, las habilidades comunicativas, que un estudiante posee, constituyen un conjunto de estrategias verbales y no verbales a través de las cuales buscan alcanzar el objetivo involucrado en el proceso de comunicación, por esa razón las estrategias metacognitivas se ajustan muy bien a esas habilidades. Por su parte, Solé (1994) señala que para hablar, escuchar, leer y escribir con eficacia, el estudiante debe formularse preguntas como ¿qué pretendo escribir?, ¿por qué?, ¿qué puedo aportar?, ¿qué dificultades tengo para escribir?, ¿cómo los puedo superar?, ¿qué significa tal palabra?, ¿habré conseguido mi propósito?, ¿tengo que corregir?, etc. Estas son preguntas que orientan al estudiante a realizar comprensivamente su 
actividad lingüística, su punto de partida es la reflexión metacognitiva sobre cómo se comunica en forma oral o escrita hasta qué recursos necesita deber usar para lograr eficacia en esa comunicación.

"Siguiendo a Martí (1999) parece que el concepto de metacognición está ligado a las estrategias, tanto en el tema de resolución de problemas en general, como en el tema del aprendizaje. Y esto por varias razones: la primera, porque si las estrategias consisten en un conjunto de acciones o procedimientos para alcanzar un fin, lo lógico es pensar que esto se lleve a cabo utilizando mecanismos reguladores y esta regulación es un aspecto de la metacognición. En segundo lugar, porque cuando se habla de estrategias muchos autores distinguen entre dos tipos de procedimientos, unos automatizados a los que llaman habilidades, técnicas, destrezas o hábitos y otros conscientes e intencionales a los que propiamente llaman estrategias" (Muñoz, 2004, p.74). Por lo tanto, en materia de metacognición se establece que el buen aprendizaje supone un proceso consciente y, por lo tanto, los estudiantes deben pasar de unos procedimientos inconscientes a otros conscientes. Esto se conseguirá a través de la explicación verbal por parte de los profesores de las estrategias de aprendizaje para que los estudiantes las incorporen a sus esquemas mentales, que dependerá de la práctica constante de los procedimientos reconstructivos de sus representaciones interiores, es decir de estrategias metacognitivas.

En cuanto a los marcadores discursivos, según muchas investigaciones de adquisición de estos elementos lingüísticos, se establece que para conocerlos y aprenderlos se necesitarán de materiales como de propuestas didácticas que los profesores tienen que innovar a partir de su experiencia. Está establecido que para que los estudiantes aprendan a usar los marcadores, el docente debe proveer casos en que se empleen distintos géneros textuales de modo que se vea expuesto a evaluar el marcador apropiado en distintas situaciones comunicativas. No se usan los mismos marcadores textuales en textos narrativos, descriptivos, expositivos $\mathrm{o}$ argumentativos. Por otro lado, se ha de tener presente que el conocimiento y aprendizaje de ciertos marcadores discursivos en relación con los géneros y secuencias textuales, implica que los estudiantes también conozcan y aprendan a usar otras partículas discursivas análogas, es decir los homólogos discursivos. Así, por ejemplo, el lingüista Antonio Messias, establece que la presencia de los marcadores "en suma y en conclusión" (clase de los reformuladores recapitulativos), en ciertos géneros textuales, pueden ser reemplazados por otros marcadores de mismo valor discursivo, como, "por ejemplo, en síntesis, en resumen", etc.

Según Portolés (1999): “Los 'marcadores del discurso' son unidades lingüísticas invariables, no ejercen una función sintáctica en el marco de la 
predicación oracional -son, pues elementos marginales-y poseen un cometido coincidente en el discurso: el de guiar, de acuerdo con sus distintas propiedades morfosintácticas, semánticas y pragmáticas, las inferencias que se realizan en la comunicación" (p.4057). Por lo tanto, los marcadores discursivos al margen de ser unidades lingüísticas invariables que guían las inferencias que se realizan en la comunicación, tienen relevancia en la comprensión del texto y deben aprenderse siguiendo estrategias lo más reflexivas posibles; en el caso de esta investigación a través de estrategias metacognitivas. Así, los marcadores discursivos influyen en la comprensión del texto que produce el lector y según Bernárdez (1995): "Es de esperar que los índices de conexión y deixis proporcionen resultados inversos: cuanto mayor sea el índice de conexión, más accesible será un texto a cualquier receptor, pues es menor la energía que deberá gastar en el procesamiento; en cambio, cuanto mayor sea el índice de deixis, mayor será la dificultad de procesamiento" (p.227). Por lo expuesto por Bernárdez, se comprende que es necesario saber emplear correctamente los marcadores discursivos de acuerdo al tipo de texto, para producir un discurso que realmente comunique lo que se desee y que el lector pueda comprenderlo con eficacia.

\section{MÉTODO}

El estudio se encuentra ubicado dentro de una investigación de tipo aplicada, porque busca solucionar problemas relacionados con el proceso de aprendizaje de los marcadores discursivos. El diseño de la investigación es experimental debido a que hubo manipulación de la variable independiente y en consecuencia modificación de la variable dependiente. Se define bajo el nivel cuasi experimental pretest - postest con grupo experimental y control; según Hernández Sampieri et al. (2010), se recurre a estos grupos cuando el investigador pretende analizar relaciones de causalidad y puede manipular la variable independiente, pero se ve obligado a partir de grupos ya formados de una manera natural, como son los grupos de estudiantes que por turnos se forman en la universidad. Este nivel de investigación con pretest - postest permite apreciar la equivalencia inicial entre los grupos y conocer los niveles de las variables dependientes antes de que se aplique el experimento y se manipule la variable independiente. Los grupos que conforman el diseño pueden observarse y luego de la etapa del experimento, observar las diferencias en la variable dependiente. Por lo tanto la variable independiente es observada durante el experimento, mientras que la dependiente es medida antes y después del experimento.

En esta investigación, se asume el enfoque mixto (cualitativo y cuantitativo). Se han obtenido resultados cualitativos narrados a partir de la observación de la influencia de la variable independiente sobre la dependiente utilizando listas 
de cotejo, focus group con entrevista, así como diarios o autoinformes de los estudiantes y del docente; mientras que se exponen resultados cuantitativos de la variable dependiente, a partir de los resultados que se obtuvieron después de aplicar el pre y post test; datos que han sido procesados a través del programa SPSS 22. Esto permitió establecer la variación entre el pre test y el pos test.

La población la constituye todos los estudiantes matriculados en Taller de Comunicación Oral y Escrita en el semestre 2015-I, que hacen un total de 1487 estudiantes y la muestra serán dos secciones de 27 estudiantes cada una, debido al tipo de investigación como a su diseño, se necesitan solo de un grupo control y otro que funcione como el grupo experimental donde se aplica el módulo innovador que incluye las estrategias metacognitivas, para el aprendizaje de los marcadores discursivos en distintas tipologías. La otra sección, que constituye el grupo control, siguió las estrategias tradicionales del taller. Estas clases constituyen grupos naturales intactos y, por lo tanto, el investigador no puede asignar aleatoriamente los sujetos a los grupos de tratamiento porque cada grupo se conforma a partir de los alumnos que pertenecen a esa clase.

El enfoque de esta investigación fue mixta por lo que se elaboraron no solo instrumentos que permiten recoger datos cuantitativos sino también instrumentos que permiten obtener datos cualitativos.

Para obtener los datos cuantitativos se aplicaron:

- Pretest y Postest: el objetivo de este test fue recoger el nivel inicial del empleo de las estrategias metacognitivas para usar los marcadores discursivos en la redacción de textos. Después de finalizado el experimento se aplicó nuevamente para analizar los cambios a través de los resultados cuantitativos. Está estructurada mediante 7 ítems complejos, albergando 17 elementos entre párrafos, léxicos y proyecto de redacción de párrafos.

Para obtener los datos cualitativos se usaron los siguientes instrumentos:

- Una lista de cotejo, un formato de entrevista y un formato de autoinforme. El objetivo de estos instrumentos fue obtener, mediante el método explicativo, la narración cualitativa del progreso de los estudiantes, utilizando la técnica de la observación y del cuestionario. Permitió observar la influencia de la variable independiente sobre la dependiente durante y al finalizar el experimento. 


\begin{tabular}{|l|l|l|l|}
\hline $\begin{array}{c}\text { Instrumento/grupo } \\
\text { Análisis cuantitiativo }\end{array}$ & \multicolumn{1}{|c|}{ Experimental } & Control & \multicolumn{1}{c|}{ Análisis } \\
\hline Pre test & I & II & $\begin{array}{l}\text { A: análisis diferencial de am- } \\
\text { bos grupos previo al experi- } \\
\text { mento. }\end{array}$ \\
\hline Post test & III & IV & $\begin{array}{l}\text { B: análisis de las diferencia en } \\
\text { las estrategias de aprendizaje } \\
\text { debidas al experimento. }\end{array}$ \\
\hline Análisis cualitativo: & $\begin{array}{l}\text { C : Evolución del } \\
\text { grupo experimental }\end{array}$ & & \\
\hline
\end{tabular}

Para manipular la variable independiente se confeccionó y utilizó un módulo experimental conformado por tres sesiones innovadoras. Se adjuntan al final de este artículo un modelo.

Las normas de la APA definen la fiabilidad como el grado en que las puntuaciones del test son consistentes, dependientes o repetibles; esto es, el grado en que están libres de los errores de medición. Por lo tanto, es importante establecer la confiabilidad de los instrumentos porque en toda investigación deben aplicarse instrumentos válidos y confiables. Una de las estrategias que se asumió para esto fue la aplicación del test a una muestra piloto antes que sean ejecutadas en la muestra total. Los resultados fueron procesados a través del alfa de Combrach, para observar así la consistencia interna del instrumento. Es necesario indicar que parte de las preguntas de los test ya fueron validadas y establecidas su confiabilidad a través de su aplicación en una investigación de mi autoría (Llontop, 2013) y para este experimento, algunos ítems de mi anterior investigación ya validados se incluyeron en el pre-test de esta tesis, otros fueron mejorados y se incrementaron algunos ítems nuevos para atender a la matriz de operacionalización. De modo los datos que se obtuvieron en su nueva aplicación, fueron confiables. Es necesario mencionar que la validez de los test como de las lista de cotejo, del formato de autoinforme y el cuestionario para las entrevistas fueron revisadas por tres expertos, doctores en educación, quienes hicieron sus observaciones oportunas para la mejora de los instrumentos.

Para el análisis estadístico de los datos se empleó el programa informático SPSS 22, utilizando la técnica de Análisis de Varianza (medias y desviaciones típicas) así como la aplicación de la T de Student con el P-valor, para contrastar las hipótesis y obtener así los resultados cuantitativos, no sin antes realizar los estudios descriptivos pertinentes. 
La $\mathrm{T}$ de Student ha permitido contrastar las hipótesis a partir de la comparación de los resultados de la aplicación del pre test respecto al post test a través del examen estadístico Kolmogorov-Smirnov (para muestras mayores de 30 individuos), test de los rangos para datos apareados de la T; que permite ver el aumento o disminución de una variable (en nuestro caso el de la variable dependiente) de modo que se observa la magnitud de cambio. Las conclusiones para cada hipótesis planteada se realizaron a través del análisis del P-Valor.

En esta investigación se tomaron en cuenta los principios jurídicos y éticos de una investigación inédita. Se respetaron los créditos u opiniones de terceros así como la propiedad intelectual de las fuentes consultadas a través de un registro de referencias de acuerdo al APA, sexta edición en inglés o tercera en español, para que se evidencie que la investigación es original. Incluso se ha citado la información que se tomó de una investigación anterior de la misma autora de esta tesis y que se relaciona con una de las variables.

\section{RESULTADOS}

Se presenta, a continuación, una serie de tablas y gráficos con los resultados obtenidos mediante la aplicación de estadísticos descriptivos e inferenciales para su respectivo análisis e interpretación, que incluye, además, la prueba de las hipótesis, procediendo de acuerdo a los objetivos de estudio.

Recolectada la información en los estudiantes de la muestra (grupo control y experimental), a través de los instrumentos de estudio como son el pre test y el pos test (para la variable dependiente) así como listas de cotejo, autoinforme, entrevista restrospectiva y elaborada (para la variable independiente), se procedió, por una parte, a la elaboración de una matriz de datos con el fin de efectuar el análisis cuantitativo y, por otra parte, a la narración de los resultados a partir de los instrumentos cualitativos. Por lo tanto, mediante el enfoque mixto, se obtuvieron los resultados que se presentan a continuación:

1) Resultados cualitativos de la variable independiente: estrategias metacognitivas

Para observar el desempeño del estudiante durante el módulo experimental, donde se aplica la variable independiente "estrategias metacognitivas", se elaboraron 3 instrumentos distintos, aparte del pretest. Estos son una lista de cotejo con escala de apreciación, un autoinforme y una entrevista a través de un cuestionario planificado. A continuación se narrarán cada resultado obtenido. 
Los resultados de la lista de cotejo con escala de apreciación con categoría cualitativa de la práctica metacognitiva, permitieron observar cómo se comportó el grupo experimental antes del experimento y cómo se presenta el grupo control en la evaluación inicial, a fin de poder comprender posteriormente los cambios que se suscitaron en el proceso de aprendizaje. De las listas de cotejo aplicadas en los 27 estudiantes del grupo experimental, se recogieron datos sobre el funcionamiento de los procedimientos metacognitivos al inicio del programa, durante y después del mismo. Se pudo observar antes de aplicar el experimento en el grupo experimental, que después de brindarles un ejercicio con marcadores discursivos y para lo cual se les dio total autonomía; estos estudiantes no aplicaron los procedimientos metacognitivos por propia iniciativa; pero sí se pudo observar que examinaron el texto, seleccionaron el marcador o trataron de encontrar el adecuado para poder construir el texto. Luego que colocaron el marcador en el texto-caso, no se observó que ejecutaran ninguna reflexión sobre la tarea específica, es decir que reflexionaran sobre qué tipo de texto debían armar, ni mucho menos sobre si existe alguna estrategia en particular para realizar dicha actividad. Después que lograron construir el texto uniendo determinadas ideas con ciertos marcadores discursivos, no autoevaluaron su producción textual, lo que no les permitió detectar sus errores y proceder a corregirlos. Se pudo observar también, que tampoco definieron el tipo de marcador de acuerdo al tipo de texto. Considero que esto ocurrió porque no tienen asimiladas todavía las estrategias metacognitivas para realizar esa actividad. Cuando finalmente concluyeron con toda la actividad, el estudiante por propia autonomía no procedió a evaluar la coherencia debido al conector que eligió, tampoco se preocupó en buscar un reemplazo o su homólogo discursivo para mejorar su texto, ni mucho menos resolver otros problemas textuales, sobre todo aquellas que corresponden al proceso de completar los marcadores según el tipo de texto (narrativo, descriptivo, expositivo y argumentativo). A la segunda semana de ejecutado el experimento, surge en ellos la capacidad para evaluar si el marcador corresponde a la tipología textual, de acuerdo al entrenamiento mediante las fichas elaboradas para las sesiones experimentales. La decisión de elegir un marcador para un tipo textual involucra mayor tiempo en la resolución del ejercicio textual.

Se pudo observar mucha mejoría en los resultados de las listas de cotejo a la mitad de la aplicación de este programa experimental con el módulo que contiene las fichas con estrategias metacognitivas, porque todos los indicadores fueron observados con regularidad. Claro está que parte del programa consiste en explicar al estudiante sobre la existencia de tales estrategias y que estarán presentes en su material de trabajo (ficha experimental) de manera recurrente, por eso deben realizar todo tipo de indicación que se da en las mismas. 
Al finalizar la aplicación del módulo y después de haber ejecutado tres fichas experimentales, se observaron todos los indicadores que aseguraban que las estrategias metacognitivas estaban presentes desde el inicio de la ejecución del ejercicio propuesto, no solo para planificar el texto, sino para desarrollarlo y evaluarlo. Se evidenció en los estudiantes mucho interés por sus materiales y mucha preocupación en seguir las estrategias metacognitivas que se hicieron explícitas a través de preguntas incluidas en la ficha experimental y que el docente en una primera etapa les leía para que las comprendan, pero a partir de la segunda sesión los estudiantes ya tuvieron autonomía. También se apreció, durante la aplicación del módulo, que las estrategias metacognitivas de planificación ejercían directa influencia sobre la adquisición (incorporación de los marcadores en su bagaje léxico) de los marcadores discursivos, que las estrategias de control de la metacognición ejercían influencia sobre la extensión (búsqueda de homólogos discursivos y/o aplicación de lo aprendido en otros textos) de los marcadores discursivos y que las estrategias de evaluación ejercían influencia sobre el refinamiento (busca corregir los errores o mal uso) de los marcadores discursivos.

Al final del experimento, los estudiantes se sintieron satisfechos porque lograron destreza en la redacción, los errores eran mínimos.

Por otra parte, los resultados de la aplicación de los autoinformes (autoevaluaciones de la actividad académica) en los estudiantes referidos a la importancia de la aplicación de las estrategias metacognitivas en sus actividades académicas, demuestran una aceptación por las mismas. Se apreció una valoración por la regulación que ejercen las estrategias metacognitivas que les ayudó en todo momento a ejecutar la tarea con mayor confianza y a no dejar pasar ningún requerimiento para llegar la meta con eficacia. Establecen que las estrategias les permiten pensar mejor, asumir las elecciones de los marcadores con mayor certeza y realizar la evaluación final de su texto para asegurar que tenga una coherencia total. Por su parte, los resultados de la entrevista retrospectiva y elaborada sobre las estrategias metacognitivas, que se aplicó a un grupo focal en forma voluntaria, dan cuenta de la relevancia del proyecto experimental para sus resultados en el taller; sobre todo en el tema de los marcadores discursivos, por cuanto les ha permitido comprender y producir mejor sus textos.

Los estudiantes establecieron que las estrategias les permitieron detectar los errores que producen cuando unen sus ideas, sobre todo a nivel de coherencia global, porque reconocieron los marcadores que se emplean en la introducción, en el desarrollo y en la conclusión de un texto. Señalaron que antes no reparaban en 
este aspecto y procedían a entregar sus productos sin mayor revisión; por lo tanto se comprueba que la aplicación de estrategias metacognitivas les permite reflexionar, controlar y evaluar sus actividades discursivas.

2) Resultados cuantitativos de variable dependiente: aprendizaje de los marcadores discursivos

Los resultados cuantitativos serán presentados de dos formas, primero, a través de un análisis intergrupo, es decir al interior de grupo control y del experimental, se presentarán los datos de normalidad y varianza a través de un test $\mathrm{T}$, luego se obtendrá el P-Valor de cada test, para realizar las comparaciones de los resultados que se obtuvieron entre el pre test y el post test.

En una segunda parte se lleva a cabo un análisis por muestras apareadas del grupo experimental, de sus resultados del pre test frente al post test. En este análisis intragrupo por cada una de las dimensiones de la variable dependiente, cada estudiante experimental es su propio control.

A) Análisis previo de los grupos antes de la intervención: estadísticos descriptivos (PRETEST)

Los resultados estadísticos de las pruebas de normalidad así como los de igualdad de varianza que dan cuenta de los casos válidos en la aplicación del pre test y pos test; para poder presentar las conclusiones a partir de esos datos, se asume el P-valor a partir de la significancia que se obtiene de la prueba de normalidad de Kolmogorov, y para concluir sobre la varianza, se asume el P-valor a partir de la significancia obtenida por la prueba varianza de Lenvene.

Se observó en el pre test tanto en el grupo control como en el experimental lo siguiente:

TABLA 1: Pruebas de normalidad pre test

\begin{tabular}{|c|c|c|c|c|c|c|c|}
\hline & \multirow{2}{*}{ grupo } & \multicolumn{3}{|c|}{ Kolmogorov-Smirnov ${ }^{\mathrm{a}}$} & \multicolumn{3}{|c|}{ Shapiro-Wilk } \\
\hline & & Estadístico & $\mathrm{gl}$ & Sig. & Estadístico & $\mathrm{gl}$ & Sig. \\
\hline \multirow{2}{*}{$\begin{array}{l}\text { Total } \\
\text { pre }\end{array}$} & control & , 143 & 27 &, 163 & 967 & 27 &, 523 \\
\hline & experimental & , 134 & 27 & ,200* & 961 & 27 & ,384 \\
\hline \multicolumn{8}{|c|}{ *. Este es un límite inferior de la significación verdadera. } \\
\hline \multicolumn{8}{|c|}{ a. Corrección de la significación de Lilliefors } \\
\hline
\end{tabular}


Podemos observar, según Kolmogorov -Smirnov (para muestras mayores a 30 individuos, entre el grupo control y experimental hacen un total 54 individuos), que la significancia del pretest para el grupo control es 0,163 y para el grupo experimental 0,200; cuando estos resultados se llevan al cuadro del P-valor para determinar las conclusiones, se establece que si el P-valor es igual o mayor que alfa $(\alpha=0,5)$ los datos provienen de una distribución normal, que es lo que se ha comprobado con esos resultados, como sigue:

P-Valor $=>\alpha$ Aceptar $\mathrm{H} 0=$ Los datos provienen de una distribución normal.

P-Valor $<\alpha$ Aceptar $\mathrm{H} 1=$ Los datos no provienen de una distribución normal.

Normalidad de calificaciones

P-Valor (control) $=0.163$

P-Valor $($ experimental $)=0.200$

$$
\begin{array}{ll}
> & \alpha=0.05 \\
> & \alpha=0.05
\end{array}
$$

Se concluye a partir de estos valores que la variable calificación (total pre test) en ambos grupos se comporta normalmente.

B) Análisis de los grupos después de la intervención: estadísticos descriptivos (PRETEST)

Después de aplicar el post test en ambos grupos (control y experimental) se concluye que los datos de la variable calificación no se comportan normalmente. Para el grupo control, en el post test se obtiene un P-valor de 0.200, mientras que el P-valor para el grupo experimental es de 0.002; por lo tanto para este grupo experimental se establece que los datos no provienen de una distribución normal. Se comprueba los cambios que se obtienen después de aplicado el experimento.

TABLA 2: Pruebas de normalidad post test

\begin{tabular}{|l|l|r|r|r|r|r|r|}
\hline \multirow{2}{*}{ grupo } & \multicolumn{3}{|c|}{ Kolmogorov-Smirnov $^{\mathrm{a}}$} & \multicolumn{3}{|c|}{ Shapiro-Wilk } \\
\cline { 3 - 8 } & & Estadístico & \multicolumn{1}{c|}{ gl } & \multicolumn{1}{c|}{ Sig. } & Estadístico & gl & \multicolumn{1}{c|}{ Sig. } \\
\hline \multirow{2}{*}{ totalpost } & control &, 129 & 27 &, $200^{*}$ &, 957 & 27 &, 310 \\
\cline { 2 - 8 } & experimental &, 219 & 27 &, 002 &, 857 & 27 &, 002 \\
\hline
\end{tabular}

*. Este es un límite inferior de la significación verdadera.

P-Valor(control) $=0.200 \quad>\quad \alpha=0.05$

P-Valor (experimental) $=0.002 \quad<\quad \alpha=0.05$

Se concluye que la variable calificación (total post test) en ambos grupos no se comporta normalmente en el post test. 
C) Contrastación de hipótesis

\section{CONTRASTACIÓN DE HIPÓTESIS GENERAL}

H1: Las estrategias metacognitivas optimizan significativamente el aprendizaje de los marcadores discursivos en estudiantes del Programa de Estudios Básicos de la Universidad Ricardo Palma, año 2015

H0: Las estrategias metacognitivas no optimizan significativamente el aprendizaje de los marcadores discursivos en estudiantes del Programa de Estudios Básicos de la Universidad Ricardo Palma, año 2015

Por el resultado del P-valor en post test del grupo control y experimental total se tiene:

Prueba de Levene (para varianzas):

$\mathrm{P}$-Valor $=>\alpha$ Aceptar $\mathrm{H} 0=$ Los varianzas son iguales

$\mathrm{P}$-Valor $<\alpha$ Aceptar $\mathrm{H} 1=$ Existe diferencia significativa entre las varianzas.

Prueba T de Student y P- Valor:

Si la probabilidad obtenida P-Valor menor o igual a $\alpha$, se rechaza la Hipótesis nula (0), se aceptar H1 ( si hay influencia)

Si la probabilidad obtenida P-Valor es mayo a $\alpha$, se acepta la Hipótesis nula (0), se rechaza la $\mathrm{H} 1$

Tabla 3: P-Valor grupo control en Postest

\begin{tabular}{|l|c|c|}
\hline \multicolumn{3}{|c|}{ P-valor grupo control en Post Test } \\
\hline P-valor (control) $=0.248$ & $>$ & $\alpha=0.05$ \\
\hline CONCLUSION: Las varianzas (total post test) en ambos grupos son iguales \\
\hline
\end{tabular}

Tabla 4: P-Valor grupo experimental en Postest

\begin{tabular}{|l|c|c|}
\hline \multicolumn{2}{|c|}{ P-valor grupo experimental en Post Test } \\
\hline $\begin{array}{l}\text { P-valor (experimental) }= \\
0.000\end{array}$ & $<$ & $\alpha=0.05$ \\
\hline $\begin{array}{l}\text { CONCLUSION: se rechaza la hipótesis nula (no optimizan el aprendizaje de marcadores) y } \\
\text { se acepta la hipótesis de investigación, que da cuenta de la influencia (sí optimizan) }\end{array}$ \\
\hline
\end{tabular}


Decisión:

Según la prueba T y el P-valor, si la probabilidad obtenida P-Valor es menor o igual a alfa, entonces se rechaza la hipótesis nula $(\mathrm{H} 0)$ y se acepta en consecuencia la Hipótesis de investigación (H1), estableciendo que sí hay influencia.

\section{CONTRASTACIÓN DE HIPÓTESIS ESPECÍFICAS}

La contrastación de las hipótesis específicas se realiza a partir de los datos obtenidos del análisis que emparejan resultados, es decir por muestras apareadas. Se presentan por dimensiones.

Dimensión 1: Adquisición del conocimiento lingüístico sobre los marcadores discursivos

H1.1 Las estrategias metacognitivas optimizan la adquisición del conocimiento lingüístico sobre los marcadores discursivos después del experimento en estudiantes del PEB de la Universidad Ricardo Palma, año 2015.

H0: Las estrategias metacognitivas no optimizan la adquisición del conocimiento lingüístico sobre los marcadores discursivos después del experimento en estudiantes del PEB de la Universidad Ricardo Palma, año 2015.

Tabla 5:Prueba de muestras emparejadas

\begin{tabular}{|c|c|c|c|c|c|c|c|c|c|}
\hline & \multicolumn{5}{|c|}{ Diferencias emparejadas } & \multirow{3}{*}{$\mathrm{t}$} & \multirow{3}{*}{ gl } & \multirow{3}{*}{$\begin{array}{c}\text { Sig. } \\
\text { (bilateral) }\end{array}$} \\
\hline & & \multirow[t]{2}{*}{ Media } & \multirow[t]{2}{*}{$\begin{array}{c}\text { Des- } \\
\text { viación } \\
\text { estándar }\end{array}$} & \multirow{2}{*}{$\begin{array}{l}\text { Media } \\
\text { de error } \\
\text { estándar }\end{array}$} & \multicolumn{2}{|c|}{$\begin{array}{l}95 \% \text { de intervalo } \\
\text { de confianza de la } \\
\text { diferencia }\end{array}$} & & & \\
\hline & & & & & Inferior & Superior & & & \\
\hline Par 1 & $\begin{array}{l}\text { Adqui- } \\
\text { sición } \\
\text { pre } \\
\text { - adqui- } \\
\text { sición } \\
\text { post }\end{array}$ & $-3,333$ & 1,981 & ,381 & $-4,117$ & $-2,550$ & $-8,745$ & 26 & ,001 \\
\hline
\end{tabular}

La significancia es menor que alfa

Sub-decisión: Según la probabilidad obtenida P-Valor (0. 001) es menor o igual a alfa, entonces se rechaza la hipótesis nula $(\mathrm{H} 0)$ y se acepta en consecuencia la Hipótesis de investigación (H1.1), estableciendo que sí hay influencia. 
Dimensión 2: extensión del conocimiento lingüístico sobre los marcadores discursivos

H1.2 Las estrategias metacognitivas optimizan la extensión del conocimiento lingüístico sobre los marcadores discursivos después del experimento en estudiantes del PEB de la Universidad Ricardo Palma, año 2015

H0: Las estrategias metacognitivas NO optimizan la extensión del conocimiento lingüístico sobre los marcadores discursivos después del experimento en estudiantes del PEB de la Universidad Ricardo Palma, año 2015

TABLA 6: Prueba de muestras emparejadas

\begin{tabular}{|c|c|c|c|c|c|c|c|c|c|}
\hline & \multicolumn{5}{|c|}{ Diferencias emparejadas } & \multirow{3}{*}{$\mathrm{t}$} & \multirow{3}{*}{ gl } & \multirow{3}{*}{$\begin{array}{l}\text { Sig. } \\
\text { (bilate- } \\
\text { ral) }\end{array}$} \\
\hline & & \multirow[t]{2}{*}{ Media } & \multirow{2}{*}{$\begin{array}{c}\text { Des- } \\
\text { viación } \\
\text { estándar }\end{array}$} & \multirow{2}{*}{$\begin{array}{l}\text { Media } \\
\text { de error } \\
\text { estándar }\end{array}$} & \multicolumn{2}{|c|}{$\begin{array}{c}95 \% \text { de intervalo } \\
\text { de confianza de la } \\
\text { diferencia }\end{array}$} & & & \\
\hline & & & & & Inferior & Superior & & & \\
\hline $\begin{array}{l}\text { Par } \\
2\end{array}$ & $\begin{array}{l}\text { Exten- } \\
\text { sión pre- } \\
\text { extensión } \\
\text { post }\end{array}$ & $-9,852$ & 1,610 & 310 & $-10,489$ & $-9,215$ & $-31,793$ & 26 & ,001 \\
\hline
\end{tabular}

- La significancia es menor que alfa

Sub-decisión: Según la probabilidad obtenida P-Valor (0. 001) es menor o igual a alfa, entonces se rechaza la hipótesis nula (H0) y se acepta en consecuencia la Hipótesis de investigación H1.2, estableciendo que sí hay influencia.

Dimensión 3: refinamiento del conocimiento lingüístico sobre los marcadores discursivos

H1.3 Las estrategias metacognitivas optimizan el refinamiento del conocimiento lingüístico sobre los marcadores discursivos después del experimento en estudiantes del PEB de la Universidad Ricardo Palma, año 2015.

H0: Las estrategias metacognitivas no optimizan el refinamiento del conocimiento lingǘstico sobre los marcadores discursivos después del experimento en estudiantes del PB de la Universidad Ricardo Palma, año 2015. 
TABLA 7: Prueba de muestras emparejadas

\begin{tabular}{|c|c|c|c|c|c|c|c|c|c|}
\hline & \multicolumn{5}{|c|}{ Diferencias emparejadas } & \multirow{3}{*}{$\mathrm{t}$} & \multirow{3}{*}{ gl } & \multirow{3}{*}{$\begin{array}{l}\text { Sig. } \\
\text { (bila- } \\
\text { teral) }\end{array}$} \\
\hline & & \multirow[t]{2}{*}{ Media } & \multirow{2}{*}{$\begin{array}{l}\text { Des- } \\
\text { viación } \\
\text { estándar }\end{array}$} & \multirow{2}{*}{$\begin{array}{l}\text { Media } \\
\text { de error } \\
\text { estándar }\end{array}$} & \multicolumn{2}{|c|}{$\begin{array}{c}95 \% \text { de intervalo } \\
\text { de confianza de la } \\
\text { diferencia }\end{array}$} & & & \\
\hline & & & & & Inferior & Superior & & & \\
\hline Par 3 & $\begin{array}{l}\text { Refina- } \\
\text { miento } \\
\text { pre } \\
\text { - refina- } \\
\text { miento } \\
\text { post }\end{array}$ & $-2,926$ & ,781 &, 150 & $-3,235$ & $-2,617$ & $-19,471$ & 26 &, 001 \\
\hline
\end{tabular}

- La significancia es menor que alfa

Sub-decisión: Según la probabilidad obtenida P-Valor (0.001) es menor o igual a alfa, entonces se rechaza la hipótesis nula ( $\mathrm{H} 0)$ y se acepta en consecuencia la Hipótesis de investigación H1.3, estableciendo que sí hay influencia.

TABLA 8: P-Valor para las tres sub hipótesis

\begin{tabular}{|c|c|c|}
\hline \multicolumn{3}{|l|}{ PRUEBA T } \\
\hline $\begin{array}{l}\text { P-Valor(1) }=0.001 \\
\text { Subhipótesis_l }\end{array}$ & $<$ & $\alpha=0.05$ \\
\hline $\begin{array}{l}\text { P-Valor }(2)=0.001 \\
\text { Subhipótesis } 2\end{array}$ & $<$ & $\alpha=0.05$ \\
\hline $\begin{array}{l}\text { P-Valor }(3)=0.001 \\
\text { Subhipótesis } 3\end{array}$ & $<$ & $\alpha=0.05$ \\
\hline \multicolumn{3}{|c|}{$\begin{array}{l}\text { CONCLUSION: } \\
\text { Se rechaza la hipótesis nula (no optimizan el aprendizaje de marcadores) y se acepta la } \\
\text { hipótesis de investigación, que da cuenta de la influencia (sí optimizan) y se confirman las } \\
\text { tres subhipótesis }\end{array}$} \\
\hline
\end{tabular}

\section{DISCUSIÓN}

La investigación tuvo como objetivo general establecer la influencia de las estrategias metacognitivas en el aprendizaje de los marcadores discursivos; objetivo que se evaluaría después de aplicar el experimento. Luego de efectuarse este, a 
través de una prueba paramétrica como es la T de Student, se evaluó la significancia y por los resultados podemos evidenciar que la hipótesis general que plantea que las estrategias metacognitivas optimizan el aprendizaje de los marcadores discursivos ha sido confirmada a través de la constatación de los resultados en los valores P-valor, que fueron menores que el valor de alfa $(\alpha=0.05)$. Por lo tanto estadísticamente los resultados fueron los esperados después del experimento. Situación similar se ha podido observar en los resultados obtenidos por cada dimensión de la variable dependiente. Los valores de la P, por cada dimensión son menores que alfa.

Esta situación hace confirmar que las estrategias metacognitivas son las ideales para que los estudiantes mejoren en su capacidad para redactar textos a nivel universitarios utilizando estos marcadores. Por los resultados se asume que las estrategias metacognitivas son adecuadas para controlar el proceso de aprendizaje eficaz; situación que también se puede apreciar en los antecedentes de estudios que involucraron estos tipos de estrategias en sus experimentos. Cuando apelamos a la metacognición, asumimos que es el mismo estudiante el responsable de la adquisición, extensión y regulación de su aprendizaje, por lo tanto se confirma que ya posee estrategias internas ideales para favorecer el entorno de aprendizaje dentro de una actividad pedagógica, a diferencia de otras estrategias que son externas y que dependen solo de la voluntad o destreza del docente.

Tal como lo sostiene Bara (2001) en su tesis doctoral Estrategias metacognitivas y de aprendizaje: estudio empirico sobre el efecto de la aplicación de un programa metacognitivo, y el dominio de las estrategias de aprendizaje en estudiantes de ESO, BUP y Universidad, cuando establece, refiriéndose a las estrategias metacognitivas, que se trata dentro de la corriente cognitiva como un camino pedagógico que persigue el objetivo de aprender a aprender, proporcionando al alumno las herramientas para que sea capaz de abordar la información y adquirir un conocimiento útil en múltiples situaciones; sosteniendo así la funcionalidad de los aprendizajes. Los resultados de nuestra investigación respecto a la de Bara están relacionados por el resultado, ya que las estrategias metacognitivas que aplicó también mejoró situaciones de aprendizaje.

Así también se establece, cuando se analizan los instrumentos cualitativos como las listas de cotejo, el autoinforme de cada estudiante y la entrevista a un grupo focal; que se comprueba la aceptación de estas estrategias por parte de los estudiantes, es decir confirman que gracias a estas estrategias ellos pudieron redactar mejor sus párrafos empleando los marcadores discursivos 
En definitiva, con este estudio, se pone en evidencia que de todas las estrategias que un docente pueda promover dentro de una sesión de clase se enfaticen a las metacognitivas, aunque esto no deja de lado otras estrategias como las cognitivas (organizan la información) y afectivas que también se pueden usar de manera complementaria. Estas estrategias se acomodan muy bien a las involucradas en esta investigación. Hay interdependencia entre las estrategias mencionadas, porque al final del experimento, a través de la entrevista que se realizó al grupo focal, se notó que los estudiantes se sintieron muy asertivos frente a este tipo de enseñanza que involucra las estrategias metacognitivas, por lo cual se deduce que sus estrategias afectivas, respecto al aprendizaje de los marcadores, mejoraron y condicionaron sus actitudes o una mejor predisposición para la actividad propuesta en los módulos.

Sin embargo, la propuesta experimental amerita para su aplicación, de una preparación exhaustiva de los docentes que desearían replicarla porque no solo se necesita que dominen el conocimiento lingüístico sobre los marcadores discursivos, sino también necesita del conocimiento de tipología textual; es importante que conozcan los tipos de marcadores que se usan en cada tipo de texto. También, al inicio del programa, se debe explicar al estudiante universitario cómo empleará la ficha de trabajo y el docente debe acompañar, todo el tiempo, el proceso de lectura de las preguntas reguladoras cognitivas incluidas en esa ficha para que los estudiantes puedan hacerlas explícitas hasta que se genere el hábito.

Los docentes tienen que ser entrenados en cómo usar las preguntas reguladoras de la metacognición en forma oral. Se necesita que se elaboren los materiales adecuados para introducir de manera transversal estas preguntas, tan igual como se muestra en las fichas experimentales que aparecen como anexos, en esta investigación. Lo que involucra disponer de mayor tiempo en la elaboración de materiales para cada sesión de aprendizaje y redefinir los planes de estudio.

Por todo lo discutido, es rentable el empleo de un tiempo adicional en el trabajo docente y en el desempeño del estudiante dentro de la práctica educativa para aplicar estas estrategias. El único problema que se presentaría como reto está en conseguir que el profesorado llegue a descubrir y tome conciencia de la necesidad del cambio e incluir esta propuesta como un proyecto de investigaciónacción para mejorar o transformar su práctica. 


\section{CONCLUSIONES}

1. El objetivo general busca evaluar la optimización que sobre el aprendizaje de los marcadores discursivos ejercen las estrategias metacognitivas y los resultados así lo han confirmado. Cuando se menciona el término de optimización hace referencia a asegurar un aprendizaje óptimo sobre todo en la redacción utilizando los marcadores, ya que para redactar adecuadamente un texto se debe confirmar la coherencia del mismo a través de los mecanismos de cohesión. Son estos mecanismos los que son ejercidos por los marcadores; por lo tanto, si para redactar se necesita la planificación del texto, donde las ideas elegidas necesitan un orden, entonces un estudiante primero debe adquirir los recursos que usará, luego necesitará organizarlos para poder textualizarlos y finalmente, después de producido el texto, tiene que evaluar si sus recursos de cohesión fueron los adecuados para el texto o tiene que cambiarlos. Por lo tanto la optimización es un conocimiento más cognitivo y procedimental que meramente teórico.

2. Los objetivos específicos fueron tres. El primer objetivo buscó evaluar la optimización de las estrategias metacognitivas en el aprendizaje de los marcadores pero a nivel de la adquisición de los marcadores, es decir que se compruebe si el estudiante tiene la capacidad de buscar, identificar y elegir o inferir el adecuado marcador para completar un texto. Este objetivo específico ha sido operacionalizado a través de una sub hipótesis que fue confirmada con los resultados estadísticos presentados anteriormente. Se confirma entonces que el grupo experimental tras el experimento ha tenido una mejora estadísticamente significativa con un P-valor de 001 , es decir casi al $100 \%$ de eficacia. Por 10 tanto la evidencia empírica recogida confirma la hipótesis.

3. El segundo objetivo específico buscó evaluar la optimización de las estrategias metacognitivas en el aprendizaje de los marcadores pero a nivel de la extensión de los marcadores, es decir que se compruebe si el estudiante después del experimento puede organizar los marcadores en el texto de acuerdo a su tipología textual, además si desarrolla el esquema textual con inclusión del marcador y finalmente si textualiza y elabora un texto adecuado. Este objetivo específico ha sido operacionalizado a través de una sub hipótesis que fue confirmada a través de los resultados estadísticos empíricos presentados.

4. El tercer objetivo específico buscó evaluar la optimización de las estrategias metacognitivas en el refinamiento de los marcadores discursivos, es decir si el estudiante pudo identificar los problemas textuales cuando no se empleaba el marcador adecuado, luego si buscó reformularlo y finalmente resolver el problema textual. Este tercer objetivo específico es el que involucra 
procedimientos más metacognitivos e intrínsecos al proceso de aprendizaje autónomo por parte del estudiante, lo que deviene en la elaboración de un texto con coherencia, cohesión y adecuación. Este objetivo específico ha sido operacionalizado a través de una sub hipótesis, que fue confirmada a través de los resultados estadísticos presentados anteriormente.

5. Ha quedado comprobada la influencia de la variable independiente sobre la variable dependiente, por lo tanto se recomienda que se elaboren módulos de aprendizaje conformados por materiales que incluyan las preguntas reguladoras de la metacognición y no solo el ejercicio o actividad. Además se necesita que el material tenga un soporte lingüístico suficiente para que el estudiante cuente con todas las herramientas gramaticales necesarias para que realice la actividad con autonomía.

6. Es necesario que el docente, que aplique estas estrategias metacognitivas en su enseñanza, requiera del conocimiento de cada marcador discursivo pero de acuerdo al tipo de texto que se redactará, por lo que es necesario la capacitación docente en cuanto a este contenido lingüístico ya que es especializado y por lo general los docentes de lengua y literatura egresan de su formación pedagógica sin una especialización adecuada.

7. Antes de comenzar la aplicación de las estrategias metacognitivas, se debe concientizar al estudiante acerca de su rol protagónico cuando se usan estas estrategias reguladoras, porque no corresponde a la enseñanza que imparte el docente, sino son inherentes al proceso de aprendizaje que genera el propio individuo.

8. Es necesaria una planificación pedagógica que evalúe los requerimientos para la aplicación de las estrategias metacognitivas, que implica contar con todos los recursos necesarios para su ejecución, considerando que el factor tiempo que se dedica a la elaboración de medios y materiales deberán ser incrementados en busca de la calidad educativa. Una vez obtenidos todos los recursos debe llevarse a cabo un entrenamiento del profesorado como aprendices y enseñantes estratégicos de estas estrategias, para que puedan realizar adecuadamente la réplica en sus estudiantes.

9. Se debe introducir esta propuesta, si así se desea, como sugieren Monereo y Solé (1999) a través de la investigación-acción guiada por especialistas psicopedagógicos, a través de una acción contextualizada, ya que no será lo mismo aplicar estas estrategias metacognitivas en educación básica que en educación superior. 
10. Se concluye también que, si se desea aplicar esta innovación en un contexto educativo determinado, se haga un diagnóstico previo de lo que demanda su ejecución y si necesita una preparación intensiva, es necesario que esta formación del profesorado como los pasos de la aplicación de la innovación se estipulen en los documentos curriculares de la institución educativa y que refleje el compromiso de su real ejecución. De esta manera la investigación pasará a ser una investigación formativa.

\section{FUENTE DE INFORMACIÓN}

\section{REFERENCIAS BIBLIOGRÁFICAS}

Bernárdez, E (1995). Teoría y epistemología del texto. Madrid: Cátedra.

Flavell, J. H. (1976). Metacognitive aspects of problem solving. In L.B. Resnick (Ed.), The nature of intelligence (pp.231-236). Hillsdale, NJ: Erlbaum

Hernández, R. et al. (2010). Metodología de la investigación (5ta. ed). México: Mac Graw Hill.

Martí, E (1999). Metacognición y estrategias de aprendizaje. En J.I. Pozo y C. Monereo (coord.). El aprendizaje estratégico. Madrid: Santillana.

Ministerio de Educación del Perú (2007). La metacognición y la comunicación. En Pedagogía Fascículo 7. Serie 1 Perú: Autor.

Monereo, C. y Solé, I. (coord.) (1999). El asesoramiento psicopedagógico: una perspectiva profesional y constructivista. Madrid: Alianza Editorial.

Muñoz, J. (2004). Enseñanza - Aprendizaje en estrategias metacognitivas en niños de educación infantil. España: Servicio de Publicaciones Universidad de Burgos.

Portolés, J y Martín, M. (1999) Los marcadores del discurso. En Bosque, I. y Demonte, V. (eds.) Gramática Descriptiva de la Lengua Española (pp. 40514213). Madrid: Espasa-Calpe.

Portolés, J. (1998). La teoría de la argumentación en la lengua y los marcadores del discurso. En Martín, M. y Montolío, E. (Eds.). Los marcadores del discurso: teoría y análisis (pp. 71-92). Madrid: Arco Libros 
Valle, et al. (1998). Las estrategias de aprendizaje: características básicas y su relevancia en el contexto escolar. En Revista de Piscodidáctica No 6. (pp.5368). Coruña: Departamento de Psicología Evolutiva

Weinstein, C. y Mayer, R. (1986). The teaching of learning strategies. En M. Wittrock (Ed.) Handbook of research on teaching. New York: Mc Millan.

\section{TESIS}

Bara, P. (2001). Estrategias metacognitivas y de aprendizaje: estudio empírico sobre el efecto de la aplicación de un programa metacognitivo, y el dominio de las estrategias de aprendizaje en estudiantes de ESO, BUP y Universidad (Tesis de doctoro inédita). Universidad Complutense de Madrid, España.

Jacinto, A. (2009). Estrategias metacognitivas y el logro de aprendizaje cognitivo biológico en alumnos de la Institución Educativa Manuel González Prada - Chimbote (Tesis de maestría inédita). USMP, Lima.

Llontop, M. (2013). Marcadores del discurso y niveles de comprensión de textos argumentativos en estudiantes de la Universidad de San Martín de Porres (Tesis de Maestría) USMP, Lima.

Llontop, M. (2015) Estrategias metacognitivas en la optimización del aprendizaje de los marcadores discursivos en estudiantes de Programa de Estudios Básicos de la Universidad Ricardo Palma, año 2015. (Tesis de Doctorado ) USMP, Lima.

Marín, F. (2010). Influencia de las estrategias metacognitivas en el desarrollo de la capacidad para solucionar problemas matemáticos (Tesis de maestría inédita). USMP, Lima.

Romero, A. (2008). Estrategias metacognitivas y desarrollo de las habilidades de comprensión lectora en los niños de 5 años (Tesis de maestría inédita). USMP, Lima.

\section{REFERENCIAS HEMEROGRÁFICAS}

Solé, I. (1994). Aprender a usar lengua. Implicaciones para la enseñanza. En Aula de innovación educativa (26), 5-10 


\section{REFERENCIAS ELECTRÓNICAS}

Nogueira da Silva, A. (2010). La enseñanza de los marcadores del discurso del español en relación con los géneros y secuencias textuales. Revista Nebrija de Lingüistica Aplicada No 9 Recuperado de http://www.nebrija.com/ revista-lingüística/files/articulosPDF/articulo_530b5dd742280.pdf 